\title{
Preemptive endovascular treatment of uncomplicated Stanford Type B aortic dissection
}

\author{
Tadashi Kitamura \\ Department of Cardiovascular Surgery, Kitasato University School of Medicine, Kanagawa, Japan \\ Correspondence to: Tadashi Kitamura. 1-15-1 Kitasato, Minami, Sagamihara, Kanagawa 252-0374, Japan. Email: funcorogash@hotmail.com. \\ Provenance: This is an invited editorial, commissioned by the Section Editor, Raffaele Giordano (Department of Advanced Biomedical Sciences, Adult \\ and Pediatric Cardiac Surgery, University of Naples Federico II, Napoli, Italy). \\ Comment on: Matsuda H. Treatment of uncomplicated type B aortic dissection. Gen Thorac Cardiovasc Surg 2017;65:74-9.
}

Submitted Jul 23, 2018. Accepted for publication Sep 21, 2018.

doi: 10.21037/jtd.2018.09.120

View this article at: http://dx.doi.org/10.21037/jtd.2018.09.120

Aortic dissection is caused by an intimal tear. Once the dissection develops, the blood flow inside the aorta becomes complicated by exchange of blood between the true and false lumina through multiple reentries, and closure of the primary tear can no longer solve all the problems. However, if there is not much risk of a rupture, and blood flow to the organs is adequate, patients normally survive the acute period without major complications. This is the reason why uncomplicated acute type B aortic dissection has been treated medically with optimal blood pressure control. However, in a certain percentage of cases, the false lumen expands, and causes aneurysmal degeneration in the chronic period (1).

The major goal for the treatment of chronic type B aortic dissection is to prevent the rupture of a dissecting aneurysm. To accomplish this goal, an either minimally invasive repair of the type B dissecting aneurysm, or prevention of aneurysmal degeneration before the false lumen expands, is required. Graft replacement of the aneurysmal segment of the dissected aorta, which is considered the current gold standard for treatment of chronic type B aortic dissection, is a direct solution to avoid rupture. However, open surgery for chronic type $\mathrm{B}$ aortic dissection requires a major thoracotomy or a thoracoabdominal incision with a cardiopulmonary bypass or a left-heart bypass, sometimes with hypothermic circulatory arrest, which is accompanied by a non-negligible mortality and morbidity (2). In addition, perfusion of the distal false lumen, which may lead to further aneurysmal degeneration downstream of the aorta, has to be taken into consideration to prevent paraplegia (3).
On the other hand, preemptive thoracic endovascular aortic repair (TEVAR) is effective in achieving aortic remodeling, and preventing an aorta-related mortality (4); however, its possible complications cannot be ignored (5). Most patients with uncomplicated type $\mathrm{B}$ aortic dissection survive the early phase without any adverse events; therefore, such a preemptive procedure has to be performed with extreme care, so as not to cause more risks than benefits.

Matsuda raised essential issues on the indication and timing of preemptive TEVAR for an uncomplicated type B aortic dissection (6). In modern medicine, the indications for treatment are influenced by evidence-based guidelines, and this evidence is often gathered from various studies; in which, the obtained data are diversely interpreted. The results of INSTEAD-XL trial (7) have been widely accepted, and have exerted a profound effect on the treatment of uncomplicated type B aortic dissection despite having inherent flaws of study design to make landmark analyses, and having a rather short observation period even when the disease has a slow growth nature. There is no doubt that invasive procedures, such as high-risk major open surgery, should be replaced by a less invasive and lowrisk endovascular treatment.

Chronic type B aortic dissection with a relatively large aortic diameter tends to develop aneurysmal degeneration in the late period (8). The maximum diameter of the dissected aorta can be easily measured on CT without a contrast enhancement. The size has always been taken into consideration to predict late aneurysmal degeneration; however, does only size matter? According to Laplace's law, 


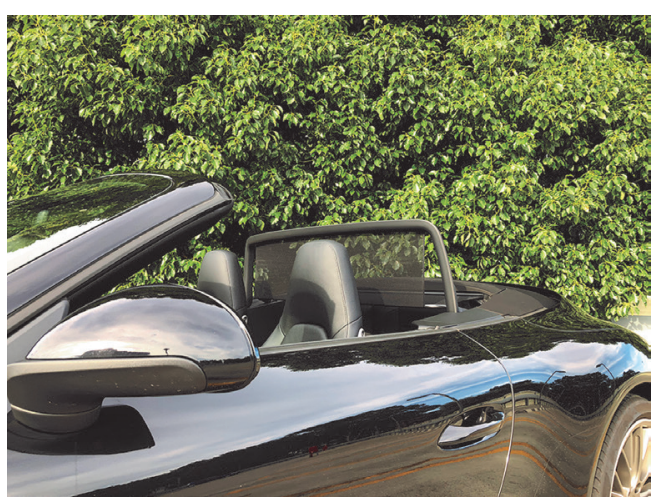

Figure 1 Mesh screen of a convertible to minimize turbulence.

wall tension is influenced by the radius of the aneurysm. In addition, wall strength, which is dependent on the thickness of the false lumen, does affect aortic enlargement; however, it cannot be measured by the usual imaging techniques at the present. Taken together, expansion of a dissecting aortic aneurysm is likely influenced by a combination of the cardiac output, blood pressure, size and location of the entry, aortic diameter, and wall thickness of the false lumen. Due to several advancements in imaging techniques, including CT and MRI, the spatial resolution of $3 \mathrm{D}$ and 4D imaging has remarkably improved, and has been utilized in daily practice for the last decade. In the future, CT may be able to show the thickness of the dissected media. In the 21 st century, artificial intelligence (AI) now plays an important role in medical diagnosis (9). Combining AI with computational fluid dynamics using software may enable rapid reading of CT imaging, identification of entry or reentries, measurement of the wall thickness of the false lumen, calculation of energy flow into the false lumen relative to the patient's specific cardiac profile, prediction of aortic growth rate, including that after primary entry closure, and recommendations on the type and size of the endovascular prosthesis. However, it is important to remember that it is the physician, not an app or AI, who actually sees and connects with the patient. It is up to the physician to comprehensively analyze the pathology, explains the risks and benefits of the options, and takes responsibility for providing the optimal treatment.

The optimal timing for preemptive TEVAR has to be discussed while taking into consideration the efficacy and procedure-related complications, which may vary among prostheses. Stent grafts with an inner frame may be advantageous for a dissected aorta, and less radial force would be better in order to avoid the risk of retrograde aortic dissection and stent graft-induced new entry (SINE) (10). During TEVAR for type B aortic dissection, the prosthesis has to be placed distally on the dissected intimo-medial wall; therefore, TEVAR should be avoided in the acute phase, when the intimo-medial wall is fragile. However, it may be reasonable to perform it in the latest period which was not classified by most studies as a chronic period (i.e., around three months after the onset). In any case, we should not forget that the radial force generated by the stent incurs the risk for SINE or stent penetration, regardless of the timing of TEVAR.

At the moment, discussions about preemptive TEVAR for uncomplicated type B aortic dissection are based on the fact that the gold standard for treatment of a chronic dissecting aneurysm is open surgery. If it is substituted by an endovascular treatment, the whole paradigm of treatment for type B aortic dissection may change. As long as there is a decent proximal landing zone, a stent graft can close the primary entry. The concept of TEVAR is different from that of open surgery; rather than simply replacing the aneurysmal portion, TEVAR includes closure of the primary entry and piling up of the stent grafts from upstream, in order to achieve a false lumen thrombosis (11). The problem is how to perfuse the branches. A number of new techniques, including branched TEVAR, fenestrated TEVAR, and candy-plug, have been reported to have acceptable short-term results (12); however, the risk for late complications, including branch occlusion and endoleak, remains a concern.

On the other hand, the effect of the damping screen to reduce turbulence (Figure 1) has been profoundly studied in the field of fluid mechanics (13). Recently, this technique has been applied in combination with endovascular treatment technologies for arterial aneurysms with branched lesions, and was shown to have promising results (14). If these new techniques, including flow-diverting multilayer stents, are able to achieve a false lumen thrombosis without compromising blood flow into the branches that come out of the false lumen, the whole paradigm of treatment of type $\mathrm{B}$ aortic dissection may change, and there may be no need for preemptive TEVAR in the subacute phase.

Type B aortic dissection expands slowly, whereas technology progresses rapidly. It would not be too advantageous to say that the current guidelines are based on insufficient long-term results from newer technologies, as well as the results of trials that were potentially biased by the discretion of patients, physicians, or manufacturers. Therefore, we must continue to accumulate more data on 
each treatment option, and keep reevaluating the risks and benefits.

In conclusion, it remains debatable as to whether preemptive TEVAR for uncomplicated type B aortic dissection is justified. Nevertheless, the stream of treatment is definitely flowing towards less invasive options.

\section{Acknowledgements}

None.

\section{Footnote}

Conflicts of Interest: The author has no conflicts of interest to declare.

\section{References}

1. Krahenbuhl E, Maksimovic S, Sodeck G, et al. What makes the difference between the natural course of a remaining type $\mathrm{B}$ dissection after type $\mathrm{A}$ repair and a primary type B aortic dissection? Eur J Cardiothorac Surg 2012;41:e110-5.

2. Preventza O, Price MD, Amarasekara HS, et al. Chronic Type I and Type III aortic dissections: a propensity analysis of outcomes after open distal repair. Eur J Cardiothorac Surg 2018;54:510-6.

3. Okita Y, Tagusari O, Minatoya K, et al. Is distal anastomosis only to the true channel in chronic type B aortic dissection justified? Ann Thorac Surg 1999;68:1586-91.

4. Nienaber CA, Kische S, Rousseau H, et al. Endovascular repair of type $\mathrm{B}$ aortic dissection: long-term results of the randomized investigation of stent grafts in aortic dissection trial. Circ Cardiovasc Interv 2013;6:407-16.

5. Nienaber CA, Rousseau H, Eggebrecht H, et al. Randomized comparison of strategies for type $\mathrm{B}$

Cite this article as: Kitamura T. Preemptive endovascular treatment of uncomplicated Stanford Type B aortic dissection. J Thorac Dis 2018;10(Suppl 33):S3862-S3864. doi: 10.21037/ jtd.2018.09.120 aortic dissection: the INvestigation of STEnt Grafts in Aortic Dissection (INSTEAD) trial. Circulation 2009;120:2519-28.

6. Matsuda H. Treatment of uncomplicated type B aortic dissection. Gen Thorac Cardiovasc Surg 2017;65:74-9.

7. Kitamura T, Torii S, Oka N, et al. Impact of the entry site on late outcome in acute Stanford type B aortic dissection. Eur J Cardiothorac Surg 2015;48:655-61; discussion 661-2.

8. van Bogerijen GH, Tolenaar JL, Rampoldi V, et al. Predictors of aortic growth in uncomplicated type B aortic dissection. J Vasc Surg 2014;59:1134-43.

9. Hirasawa T, Aoyama K, Tanimoto T, et al. Application of artificial intelligence using a convolutional neural network for detecting gastric cancer in endoscopic images. Gastric Cancer 2018;21:653-60.

10. Ma T, Dong $\mathrm{ZH}, \mathrm{Fu} \mathrm{WG}$, et al. Incidence and risk factors for retrograde type A dissection and stent graft-induced new entry after thoracic endovascular aortic repair. J Vasc Surg 2018;67:1026-33.

11. Girish A, Padala M, Kalra K, et al. The Impact of Intimal Tear Location and Partial False Lumen Thrombosis in Acute Type B Aortic Dissection. Ann Thorac Surg 2016;102:1925-32.

12. Kamman AV, de Beaufort HW, van Bogerijen GH, et al. Contemporary Management Strategies for Chronic Type B Aortic Dissections: A Systematic Review. PLoS One 2016;11:e0154930.

13. Groth J, Johansson AV. Turbulence reduction by screens. J Fluid Mech 1988;197:139-55.

14. Sfyroeras GS, Dalainas I, Giannakopoulos TG, et al. Flowdiverting stents for the treatment of arterial aneurysms. J Vasc Surg 2012;56:839-46.

(English Language Editor: Jeremy Dean Chapnick, AME Publishing Company) 